\title{
Escala de Necessidade de Pertencimento: Adaptação e Evidências de Validade
}

\author{
Camila Azevedo Gastal - Universidade de Brasilia, Brasilia, Brasil \\ Ronaldo Pilati - Universidade de Brasilia, Brasilia, Brasil
}

\begin{abstract}
Resumo
Seres humanos possuem uma necessidade fundamental de pertencer a um grupo. Tal necessidade de pertencimento possui uma dimensão disposicional, de forma que indivíduos diferem quanto à motivação pela procura de conexões sociais e o quanto valorizam ser aceitos pelas outras pessoas. A Escala de Necessidade de Pertencimento (ENP) é um instrumento elaborado para medir tal dimensão individual. O objetivo deste trabalho foi adaptar e produzir evidências de validade da ENP para o contexto brasileiro. Foram realizados dois estudos com amostras independentes. Análises fatoriais exploratórias e confirmatórias apontaram para uma estrutura unifatorial com características psicométricas semelhantes às da escala original, indicando que a ENP pode ser considerada adequada para uso no contexto brasileiro. Em análises de convergência a ENP correlacionou-se positivamente com empatia. Ressalva-se que mais estudos são necessários para a determinação de sua estrutura fatorial, além de pesquisa transcultural sobre a necessidade de pertencimento.

Palavras-chave: necessidade de pertencimento, conexão social, avaliação psicológica
\end{abstract}

The Need to Belong Scale: Adaptation and Evidences of Validity

\begin{abstract}
Human beings have a fundamental need to belong to groups. That need has a dispositional dimension, as individuals differ on how strong is their motivation to search social connections and how much they value being accepted by others. The Need to Belong Scale (NTBS) is an instrument elaborated to measure such individual dimension of the need to belong. The present research aimed to adapt and collect validity evidence for the NTBS to the Brazilian context. The research was composed of two studies with independent samples. Factor analysis, exploratory and confirmatory, pointed to a unifactor solution with psychometric properties similar to the ones of the original scale, indicating that the NTBS may be adequate for use in the Brazilian context. In convergence analysis the NTBS was positive correlated with an empathy measure. We highlight the need for more studies to define the unifactor structure. We also suggest cross-cultural comparisons concerning the need to belong.

Keywords: need to belong, social connection, psychological evaluation
\end{abstract}

Escala de Necesidad de Pertenencia: Adaptación y Evidencias de Validez

\begin{abstract}
Resumen
Los seres humanos poseen una necesidad fundamental de pertenecer a un grupo y esa necesidad tiene una dimensión disposicional, de modo que los individuos difieren en términos de motivación buscando conexiones sociales, y cómo valorizar ser aceptados por los demás. La Escala de Necesidad de Pertenencia (ENP) es un instrumento para medir tal dimensión individual, el objetivo de este trabajo fue adaptar y reunir evidencias de validez de la ENP en el contexto brasileño y fueron realizados dos estudios con muestras independientes. Los análisis factoriales, exploratorios y confirmatorios, señalaron una estructura unifactorial con características psicométricas similares a la escala original, indicando que la ENP puede ser considerada adecuada para su uso en el contexto brasileño. En análisis de convergencia la ENP se correlacionó positivamente con la empatía. Destacamos la necesidad de más estudios para la determinación de su estructura factorial, además de investigaciones transculturales sobre la necesidad de pertenencia.

Palabras clave: necesidad de pertinência, conexión social, evaluación psicológica
\end{abstract}

É sabido que a espécie humana vive naturalmente em grupos, de forma que as chances de sobrevivência em sua história evolutiva foram maiores para indivíduos ligados socialmente. Baumeister e Leary (1995) argumentam que os seres humanos possuem uma necessidade básica por pertencimento, o que motiva a busca por relações sociais profundas e positivas. De acordo com os autores, tal necessidade é fundamental, básica para uma vida saudável e satisfatória e está presente em todos os indivíduos. Além disso, a não satisfação de tal necessidade está intimamente relacionada a consequências negativas, tanto psicológicas

Disponivel em wnw.scielo.br quanto fisiológicas (Cacioppo, Hawkley \& Thisted, 2010; Cornwell \& Waite, 2009). Por estar relacionada a diversas variáveis psicológicas relevantes, torna-se necessário o levantamento de evidências de validade de um instrumento que avalie tais motivações e permita que o construto seja relacionado com outros aspectos da vida social humana. Este estudo teve como objetivo adaptar e produzir evidências de validade, para o contexto brasileiro, de um instrumento amplamente utilizado na literatura internacional que avalia a necessidade de pertencimento (Leary, Kelly, Cottrell, \& Schreindorfer, 2013).

http://dx.doi.org/10.1590/1413-82712016210206 
Necessidade de Pertencimento (Need to belong)

A necessidade de pertencimento foi primeiramente mencionada por Baumeister e Leary (1995), sendo definida como uma motivação que seres humanos têm para procurar e manter laços socais profundos, positivos e recompensadores. Dessa forma, ela se refere não só à necessidade de estar inserido em um grupo, mas à qualidade dos laços estabelecidos com outros indivíduos e o sentimento de aceitação presente.

Como os próprios autores apontam, a maior contribuição da teoria da necessidade de pertencimento não foi unicamente apontar a existência de tal necessidade, mas sim salientar seu poder de influência sobre outras variáveis psicológicas e fisiológicas (Baumeister, 2012). Um estudo de Begen e Turner-Cobb (2011) mostrou que a percepção de pertencimento está ligada à incidência de humores positivos e a uma menor incidência de sintomas fisiológicos de doenças. Os efeitos positivos de aceitação social e pertencimento são observados em indivíduos classificados com estilo de apego evitativo, o que reforça o caráter fundamental e geral de tal motivação (Carvalho \& Gabriel, 2006). A caracterização de tal construto como uma necessidade implica que a não satisfação dela é prejudicial para o indivíduo (Baumeister, 2012). Déficits no sentimento de pertencimento foram identificados como prejudiciais às habilidades de socialização, levando à ansiedade e isolamento social (Baumeister, Brewer, Tice, \& Twenge, 2007; Brown, Silvia, Myin-Germeys, \& Kwapil, 2007; DeWall \& Baumeister, 2006). Além disso, o sentimento de solidão tem valor preditivo em relação à mortalidade entre adultos mais velhos (Tilvis et al., 2012) e está relacionada com decréscimos na saúde física e habilidades cognitivas (Hawkley \& Cacioppo, 2010).

A necessidade de pertencimento tem sido investigada tanto como uma variável situacional como uma característica individual. Em uma perspectiva situacional, DeWall, Baumeister e Vohs (2008) manipularam o sentimento de pertencer comunicando aos participantes que os testes respondidos indicavam um futuro de pertencimento, aceitação e relações sociais positivas. É também possível encontrar na literatura a utilização de práticas de meditação que eliciam sentimentos de conectividade social (Waytz \& Epley, 2012).

Apesar de ser definida como uma motivação comum a todos os seres humanos, existe variação na força e influência da necessidade de pertencimento na vida de cada indivíduo, o que indica a importância de compreender esse fenômeno também a partir de uma perspectiva disposicional. Alguns indivíduos são fortemente motivados a manter relações de aceitação e pertencimento e direcionam maiores esforços para tal objetivo, enquanto outros tem uma motivação mais fraca para manter conexões sociais (Leary \& Kelly, 2009).

Entre os instrumentos utilizados para mensurar diferenças individuais na necessidade de pertencimento, a Escala de Necessidade de Pertencimento (ENP) foi primeiramente publicada por Leary, Kelly, Cottrell e Schreindorfer (2013), apresentada como um instrumento que visa medir a motivação individual pela procura de conexões sociais e o quanto o indivíduo valoriza ser aceito pelas outras pessoas (Leary \& Kelly, 2009). A escala possui 10 itens agrupados em uma estrutura unifatorial. A publicação da escala contou também com nove estudos correlacionando a ENP a medidas de outros construtos referentes ao grau em que as pessoas desejam procurar por interações sociais e estabelecer laços. Correlações fracas à moderadas foram observadas entre a ENP e medidas de extroversão $(r=0,16 ; p<0,05)$, sociabilidade $(r=0,32 ; p<0,01)$ e necessidade por afiliação $(r=0,26 ; p<0,01)$. Apesar dos autores afirmarem que tais resultados reforçam o caráter discriminante da escala em relação a esses construtos, aponta-se como limitação a falta de avaliações de validade convergente no estudo original. Além disso, propriedades psicométricas da escala foram discutidas referindo-se apenas aos índices de confiabilidade encontrados nas 15 amostras avaliadas, que variaram de 0,78 a 0,87 .

A necessidade de pertencimento individual influencia como o sujeito percebe e se comporta no meio social. A valorização da aceitação e a necessidade de estabelecer laços tornam os indivíduos mais bem adaptados para operar no meio social. Indivíduos com scores altos na ENP apresentam maior sensibilidade para pistas sociais, de forma que são mais bem-sucedidos em realizar avaliações acuradas de emoções de outros indivíduos e identificar situações de rejeição (Carvalho \& Pelham, 2006; Pickett, Gardner, \& Knowles, 2004). Tais indivíduos também apresentam mais comportamentos socialmente desejáveis que contribuem para manutenção da positividade das relações e preservação de laços afetivos, como cooperação (De Cremer \& Leonardelli, 2003).

Variações de intensidade na necessidade de pertencimento são ainda pouco exploradas de um ponto de vista transcultural. Espera-se, com o presente estudo, contribuir para a instrumentalização da avaliação da necessidade de pertencimento na população brasileira e possibilitar estudos futuros que tratem do fenômeno, entre eles aqueles que busquem comparar 
transculturalmente a necessidade de pertencimento. Existem aspectos teóricos suficientes na área para se argumentar que diferentes culturas e ambientes sociais podem alterar as formas como tal motivação se manifesta e afeta comportamentos e atitudes, o que demanda estudos futuros que busquem explorar tais efeitos.

Este trabalho teve como objetivo adaptar para o contexto brasileiro e avaliar as qualidades psicométricas da Escala de Necessidade de Pertencimento (ENP). Para alcançar tal objetivo foram realizados dois estudos com amostras independentes. O Estudo 1 teve por objetivo traduzir a escala e avaliar a estrutura fatorial do instrumento por meio de análises fatoriais exploratórias e o Estudo 2 visou replicar a estrutura fatorial encontrada no Estudo 1 por meio de análises fatoriais confirmatórias.

Estudo 1

\section{Método}

\section{Participantes}

Participaram do estudo 159 indivíduos (106 mulheres) sendo sua maioria estudantes universitários (137 de nível superior incompleto). A média de idade foi de 20,65 anos $(D P=3,24)$.

\section{Instrumentos e Materiais}

Escala de Pertencimento - A escala utilizada foi uma versão traduzida da escala original publicada por Leary et al. (2013), com 10 itens $(\alpha=0,78)$. Foi utilizado o método de tradução-retradução (Hambleton \& Zeniski, 2011). A partir da comparação das duas versões (traduzida e retraduzida) foram feitos ajustes e chegou-se a versão final traduzida. $\mathrm{O}$ enunciado do instrumento orientava o sujeito a indicar o quanto as frases listadas eram verdadeiras para ele, utilizando uma escala de cinco pontos (1 - nem um pouco, 2 - levemente, 3 - moderadamente, 4 - muito e 5 - extremamente).

\section{Empatia - Interpersonal Reactivity Index} - Foram utilizadas duas subescalas do instrumento adaptado por Sampaio, Guimarães, Santos Camino, Formiga e Menezes, (2011). A primeira foi de Consideração Empática $(\alpha=0,80)$, composta de 7 itens como "Incomodo-me com as coisas ruins que acontecem com os outros". A segunda foi de Tomada de Perspectiva $(\alpha=0,75)$, composta por 6 itens, como "Escuto os argumentos dos outros, mesmo estando convicto da minha opinião". O participante respondia o quão bem as sentenças o descreviam em uma escala de cinco pontos cujos extremos eram 1 - não me descreve bem e 5 descreve-me muito bem.

PANAS - Afetos Positivos - Os participantes responderam aos nove adjetivos de humor positivo $(\alpha$ $=0,80)$ da versão traduzida da Positive and Negative Affect Schedule (PANAS) (Carvalho et al., 2013). O sujeito indicava o quanto ele normalmente sentia as emoções listadas, em uma escala de cinco pontos $(1-$ nem um pouco, 2 - raramente, 3 - ocasionalmente, 4 - frequentemente e 5 - bastante)

Socialização - Modelo dos Cinco Grandes Fatores - Foram utilizados os marcadores reduzidos de socialização do modelo dos Cinco Grandes Fatores, com cinco itens $(\alpha=0,69)$ (Filho, Machado, Teixeira, $\&$ Bandeira, 2012). O participante assinalava em uma escala de cinco pontos (1 - nem um pouco, 2 - um pouco, 3 - mais ou menos, 4 - muito e 5 - muitíssimo) o quanto as características listadas o descreviam bem.

\section{Procedimento}

A coleta foi realizada presencialmente em salas de aula de uma universidade. A primeira parte do questionário esclarecia questões éticas a respeito da pesquisa e solicitava o consentimento escrito do participante. A apresentação dos instrumentos tinha a seguinte ordem: medida de socialização, ENP, medida de empatia, PANAS e questionário de dados sociodemográficos. O software utilizado na análise de dados foi o IBM SPSS, onde foram conduzidas análises de componentes principais, análises de fatoração do eixo principal e correlações bivariadas.

\section{Resultados e Discussão}

A estrutura da ENP foi primeiramente analisada por meio da análise de componentes principais e pela inspeção visual do gráfico de sedimentação (screeplot). A amostra se mostrou adequada para a análise dos componentes principais com $\mathrm{KMO}=0,78$.

A análise de componentes foi realizada sem rotação ou especificação de componentes a serem extraídos, indicando três componentes com autovalor superior a 1. Um único fator explicava $35,49 \%$ da variância. A adição do segundo fator em diante apresenta um baixo ganho no nível de variância explicada. É necessário ressaltar que tal critério tende a superestimar o número de componentes a serem extraídos, sendo a análise paralela 
o critério de decisão apontado como menos suscetível à superestimação e também o mais preciso (Laros, 2005).

A análise paralela foi realizada utilizando uma plataforma na internet, elaborada para tal fim (Patil, Surendra, Singhm, \& Donavan, 2007). Foram geradas 100 matrizes aleatórias com valores randômicos e foram extraídos autovalores dessas matrizes, com a média de 95\% dos eigenvalues aleatórios. Os valores da matriz de correlação dos dados empíricos foram então comparados com os autovalores gerados a partir das matrizes randômicas. Os dois primeiros autovalores randômicos gerados foram 1,40 e 1,27 . Os dois primeiros valores da matriz de correlação dos dados foram 3,54 e 1,22. Percebe-se, assim, que o valor da primeira dimensão excede o autovalor gerado randomicamente. A partir da segunda dimensão, nenhum outro valor derivado dos dados empíricos excedeu os valores randômicos. Levando em consideração o índice de variância explicada por uma dimensão única e a análise paralela, optou-se pela extração de um único fator.

Tal decisão é congruente com a estrutura fatorial da escala original, permitindo uma melhor comparação de propriedades psicométricas e escores obtidos entre a versão original e a versão adaptada. Na solução unifatorial, os itens apresentaram cargas que variaram de 0,72 a 0,22 , sendo que apenas o item 7 apresentou carga abaixo de 0,30 . Itens completos e suas respectivas cargas fatoriais são apresentados na Tabela 1.

O índice de consistência interna (alfa de Cronbach) foi de 0,78 , e a retirada do item 7 não resultava em aumento considerável desse valor. As correlações entre a ENP e outros instrumentos incorporados à coleta (Socialização, IRI e PANAS) se mostraram não significativas, com exceção da subescala de Consideração Empática, que apresentou $r=0,18 ; p=0,02$. Estudos anteriores encontraram correlações baixas e significativas entre a ENP e o fator de afetos positivos da PANAS e medidas de Socialização (Nichols \& Webster, 2013; Leary et al., 2013), ao contrário do que foi observado na presente amostra.

\section{Estudo 2}

\section{Método}

\section{Participantes}

Participaram do estudo 166 indivíduos (108 mulheres), com média de 23,69 anos ( $D P=9,98)$, sendo a maioria estudantes universitários (126 com nível superior incompleto).

\section{Instrumentos e Materiais}

Os instrumentos utilizados foram os mesmos do Estudo 1.

\section{Procedimento}

A coleta foi realizada por meio de uma plataforma de survey on-line (EFS). Os participantes consentiam com a participação e procediam para responder os instrumentos. A ordem de apresentação dos instrumentos era randomizada, permanecendo constante apenas o consentimento ao início da coleta e o questionário de

Tabela 1

Cargas Fatoriais Para cada Item da Solução Unifatorial da Escala de Necessidade de Pertencimento (ENP)

\begin{tabular}{lc}
\hline & Carga \\
& Fatorial \\
\hline 10. Meus sentimentos são facilmente feridos quando eu sinto que outras pessoas não me aceitam. & 0,72 \\
5. Eu quero que outras pessoas me aceitem. & 0,71 \\
9. Me incomoda muito quando eu não sou incluído nos planos das outras pessoas. & 0,63 \\
8. Eu tenho uma forte necessidade de pertencimento a outras pessoas. & 0,61 \\
4. Eu preciso sentir que existem pessoas com quem eu posso contar em momentos de necessidade. & 0,49 \\
6. Eu não gosto de ficar sozinho. & 0,45 \\
3. Eu raramente me preocupo se outras pessoas se importam comigo. & $0,45^{*}$ \\
1. Se outras pessoas não parecem me aceitar, eu não deixo que isso me aborreça. & $0,43^{*}$ \\
2. Eu me esforço para não fazer coisas que vão fazer outras pessoas me evitarem ou me rejeitarem & 0,43 \\
7. Ficar distante dos meus amigos por longos períodos de tempo não me incomoda. & $0,22^{*}$
\end{tabular}

* Itens invertidos 
dados sociodemográficos ao final. A análise dos dados foi conduzida com o software AMOS, a partir do qual foram realizadas análises fatoriais confirmatórias, testando a adequação de um modelo unifatorial. Os índices observados para avaliação do ajuste do modelo foram $\mathrm{X}^{2}$; CFI (Comparative Fit Index); RMSEA (Root Mean Square ErrorofAproximation); NFI (Normed Fit Index), GFI (Goodness-of-fit-Index) e TLI (Tucker-Lewis Index). Os valores de cada índice adotados como critério de ajuste foram: CFI superior a 0,90 ; RMSEA próximo ou inferior a 0,08 ; GFI superior a 0,90 ; NFI superior a 0,90 e TLI próximo ou superior a 0,95 . O método de estimação utilizado na análise fatorial confirmatória foi o Maximum Likelihood (MLE). Os casos ausentes foram substituídos pela média da amostra. O coeficiente de normalidade multivariada (Mardia, 1977) foi igual a 3,15 , sendo um valor $<5$ indicativo de normalidade (Byrne, 2010).

\section{Resultados e Discussão}

A escala também apresentou índice de confiabilidade satisfatório na amostra coletada no Estudo 2, $\alpha$ $=0,82$. A partir da substituição dos casos ausentes e da constatação de normalidade multivariada suficiente, procedeu-se com a análise confirmatória da estrutura unifatorial por meio do método MLE. Obteve-se o valor de $\mathrm{X}^{2}=109,875, \mathrm{GL}=35$ e $p<0,001$. A análise mostrou índices de GFI $=0,875, \mathrm{CFI}=0,860$, NFI $=0,811$, TLI $=0,82$ e RMSEA $=0,114$. Tais índices de adequação do modelo são classificados como pouco satisfatórios. Foram encontradas correlações bivariadas significativas entre a ENP e a subescala de consideração empática $(r=0,24, p<0,01)$ e a medida de Socialização $(r=0,19 ; p=0,01)$.

Uma inspeção visual da matriz de resíduos padronizados revelou apenas dois valores considerados altos (> 2,58) (Byrne, 2010). Esses valores se referiam à correlação entre os itens 7 e 3 da escala, cujo valor de resíduo foi de 2,74 , e à correlação entre os itens 3 e 1 , com valor de resíduo de 3,80. Cargas fatoriais de cada item obtidas na análise fatorial confirmatória são exibidas na Tabela 2.

A análise dos índices de modificação evidenciou que existem correlações elevadas entre os erros dos itens 2 e 9, e entre os itens 1 e 3 . Correlações muito elevadas podem indicar deficiência no valor discriminante dos itens mencionados na escala ou a presença de dimensões adicionais não estimadas.
Tabela 2

Cargas Fatoriais da ENP Obtidas pelo MLE

\begin{tabular}{lcc}
\hline & $\mathrm{CP} *$ & $\mathrm{RC}$ \\
\hline Item 3 & 0,373 & 3,07 \\
Item 1 & 0,313 & $-* *$ \\
Item 7 & 0,154 & 1,70 \\
Item 2 & $-0,538$ & $-3,52$ \\
Item 4 & $-0,588$ & $-3,61$ \\
Item 6 & $-0,609$ & $-3,64$ \\
Item 9 & $-0,710$ & $-3,77$ \\
Item 5 & $-0,737$ & $-3,79$ \\
Item 8 & $-0,745$ & $-3,80$ \\
Item 10 & $-0,838$ & $-3,87$ \\
\hline
\end{tabular}

*CP - Coeficiente Padronizado de Estimação; RC - Razão Crítica da Estimação.

** Esse coeficiente foi fixado para permitir a estimação dos demais parâmetros do modelo.

\section{Considerações Finais}

O presente trabalho teve como objetivo adaptar e produzir evidências de validade da Escala de Necessidade de Pertencimento (The Need to Belong Scale - Leary et al., 2013). Foram conduzidas análises fatoriais confirmatórias e exploratórias para avaliar as propriedades psicométricas da escala. Análises fatoriais exploratórias e a análise paralela apontaram para uma estrutura unifatorial no Estudo 1. Porém, o Estudo 2 apontou para uma adequação pouco satisfatória dessa estrutura. Apesar dos resultados observados no Estudo 2, optou-se por manter a estrutura considerando que a possibilidade de acréscimo de outro componente não seria embasada teoricamente. Além disso, manter tal estrutura confere valor comparativo entre a presente versão da ENP e outras versões, o que é relevante para estudos transculturais futuros sobre o tema.

Como limitações do presente estudo, aponta-se os índices de ajuste obtidos na análise fatorial confirmatória para o modelo unifatorial, posicionados abaixo do adequado. Sugere-se a redução embasada em uma justificativa teórica do número de itens na escala como uma possibilidade de aprimorar seus indicadores em tal análise, considerando que foram observadas altas correlações entre itens a partir da inspeção dos índices de modificação. Talvez, futuramente, uma possibilidade 
de aprimoramento da versão brasileira da ENP seja a redução de seus itens, mas considera-se essa iniciativa prematura no momento. Encoraja-se que mais estudos sejam feitos visando o aprimoramento da versão, a fim de identificar possíveis modificações que possam levar à melhora dos índices apontados aqui como não satisfatórios.

As correlações positivas encontradas entre a ENP e a subescala de Consideração Empática e de Socialização, apesar de fracas, corroboram a premissa de que indivíduos que valorizam o estabelecimento de relações são melhor adaptados para agir no meio social, desempenhando comportamentos desejáveis que incentivem interações positivas, principalmente percebendo e considerando as emoções de outros indivíduos (Leary \& Kelly, 2009).

Ressalta-se que não existem na literatura publicações tratando de análises fatoriais realizadas no instrumento, sendo os dados apresentados aqui de importância comparativa para futuras avaliações psicométricas do instrumento. Apesar de se tratar de um construto teoricamente bem formulado e definido no campo da psicologia social (Leary et al., 2013), sugere-se avaliações psicométricas mais extensivas da ENP, tanto em amostras brasileiras quanto de outros países, de forma que seja possível estender os conhecimentos acerca das características da escala.

A versão adaptada da escala apresentou características satisfatórias considerando critérios como confiabilidade, validade e praticidade (Boyle, Saklofke e Matthews, 2015). Índices como o alfa de Cronbach e as avaliações fatoriais realizadas se reportam aos dois primeiros critérios. Já o número de itens da escala confere praticidade à sua aplicação. Logo, apesar das limitações aqui apontadas, é possível argumentar que a versão adaptada para o Brasil já pode ser utilizada. Porém, é necessário considerar que a presente versão ainda necessita de ajustes para otimizar sua estrutura e alcançar indicadores mais robustos.

Além de ressaltar a necessidade de mais estudos referentes às propriedades da escala, também se aponta como direções futuras de pesquisa em necessidade de pertencimento a avaliação da ENP em diferentes culturas. Alguns estudos já avaliaram como diferentes culturas estão relacionadas a diferentes percepções do que é pertencer e à sensibilidade à exclusão social (Pfundmair et al., 2015; Pfundmair, Graupmann, Frey, \& Aydin, 2015; Schmitt, 2010). Trata-se de um componente determinante para a compreensão de como se estabelecem as relações sociais, sendo a avaliação transcultural de constructos da psicologia social de extrema relevância para a área (Chiu, Chia, \& Wan, 2015). Dessa forma, o presente estudo contribui também para a instrumentalização para futuras avaliações transculturais da necessidade de pertencimento no Brasil.

$\mathrm{O}$ estudo da necessidade de pertencimento perpassa questões a respeito da natureza humana, cultura, emoções e relações interpessoais. As investigações envolvendo tal necessidade no contexto brasileiro estão ainda em fase inicial, o que torna essencial a instrumentalização que possibilite seu desenvolvimento.

\section{Referências}

Baumeister, R. F., Brewer, L. E., Tice, D. M., \& Twenge, J. M. (2007). Thwarting the Need to Belong: Understanding the Interpersonal na Inner Effects of Social Exclusion. Social and Personality Compass, 1(1), 506-520. doi: 10.1111/j.1751-9004.2007.00020.x

Baumeister, R. F., \& Leary, M. R. (1995). The need to belong: Desire for interpersonal attachments as a fundamental human motivation. PsychologicalBulletin, 117(3), 497-529. doi: 10.1037/0033-2909.117.3.497

Baumeister, R. F. (2012). Need-to-belong theory. Em Van Lange, P. A. M., Kruglanski, A. W. \& Higgins, E. T. (Eds.), Handbook of theories of social psychology (pp. 121-140). London: Sage.

Begen, F. M., \& Turner-Cobb, J. M. (2011). The need to belong and symptoms of acute physical health in early adolescence. Journal of Health Psychology, 0(0), 10.doi: $0.1177 / 1359105311431176$

Boyle, G. J., Saklofske, D. H. \& Matthews, G. (2015). Em Boyle, G. J., Saklofske, D. H. \& Matthews, G. (Eds) Measures of Personality and Social Psychological Constructs (pp.3-15). London: Elsevier

Brown, L. H., Silvia, P. J., Myin-Germeys, I., \& Kwapil, T. R. (2007). When the need to belong goes wrong: the expression of social anhedonia and social anxiety in daily life. Psychological Science, 18(9), 778-782. doi: 10.1111/j.1467 9280.2007.01978.x

Byrne, B. M. (2010). Structural equation modeling with AMOS: Basic concepts, applications, and programming. New York: Routledge.

Cacioppo, J. T., Hawkley, L. C., \& Thisted, R. A. (2010). Perceived social isolation makes me sad: 5 -year cross-lagged analyses of loneliness and depressive symptomatology in the Chicago Health, Aging, and Social Relations Study. Psychology and aging, 25(2), 453. doi: $10.1037 / \mathrm{a} 0017216$ 
Carvalho, H. W. D., Andreoli, S. B., Lara, D. R., Patrick, C. J., Quintana, M. I., Bressan, R. A.,... \& Jorge, M. R. (2013). Structural validity and reliability of the Positive and Negative Affect Schedule (PANAS): Evidence from a large Brazilian community sample. Revista Brasileira de Psiquiatria, 35(2), 169-172. doi:10.1590/1516-4446-2012-0957

Carvallo, M., \& Gabriel, S. (2006). No man is an island: The need to belong and dismissing avoidant attachment style. Personality and Social Psychology Bulletin, 32(5), 697-709. doi: 10.1177/0146167205285451

Carvallo, M., \& Pelham, B. W. (2006). When fiends become friends: The need to belong and perceptions of personal and group discrimination. Journal of Personality and Social Psychology, 90(1), 94. doi: 10.1037/0022-3514.90.1.94

Chiu, C., Chia, S., \& Wan, W. W. N., (2015). Em G. J. Boyle, D. H. Saklofske \& G. Matthews, (Eds) Measures of Personality and Social Psychological Constructs (pp. 621-651). London: Elsevier

Cornwell, E. Y., \& Waite, L. J. (2009). Social disconnectedness, perceived isolation, and health among older adults. Journal of Health and Social Behavior, 50(1), 31-48. doi: 10.1177/002214650905000103

De Cremer, D., \& Leonardelli, G. J. (2003). Cooperation in social dilemmas and the need to belong: The moderating effect of group size. Group Dynamics: Theory, Research, and Practice, 7(2). doi: 10.1037/1089-2699.7.2.168

DeWall, C. N., \& Baumeister, R. F. (2006). Alone but feeling no pain: Effects of social exclusion on physical pain tolerance and pain threshold, affective forecasting, and interpersonal empathy. Journal of personality and social psychology, 91(1). doi: 10.1037/0022-3514.91.1.1

DeWall, C. N., Baumeister, R. F., \& Vohs, K. D. (2008). Satiated with belongingness? Effects of acceptance, rejection, and task framing on self-regulatory performance. Journal of personality and social psychology, 95(6). doi: 10.1037/a0012632

Filho, N. H., Machado, W. L, Teixeira, M. A. P., \& Bandeira, D. R. (2012). Evidências de validade de marcadores reduzidos para a avaliação da personalidade no modelo dos cinco grandes fatores. Psicologia: Teoria e Pesquisa, 28(4), 417-423. doi: 10.1590/S0102-37722012000400007
Hambleton, R. K., \& Zenisky, A. L. (2011). Translating and adapting tests for cross cultural assessments. Em D. Matsumoto \& F. J. R. van de Vijver (Eds.). Cross Cultural Research Methods in Psychology (pp. 46-70). New York: Cambridge.

Hawkley, L. C., \& Cacioppo, J. T. (2010). Loneliness matters: A theoretical and empirical review of consequences and mechanisms. Annals of Behavioral Medicine, 40(2), 218-227. doi: 10.1007/ s12160-010-9210-8

Laros, J. A. (2005). O uso de análise fatorial: Algumas diretrizes para pesquisadores. Em Pasquali, L. (Ed.), Análise fatorial para pesquisadores (pp. 163184). Brasília: LabPAM.

Leary, M., R., \& Kelly, K. (2009). Em M. R. Leary \& R. H. Hoyle. Handbook of Individual differences in social behavior. (pp. 400-409). New York: Guildford.

Leary, M. R., Kelly, K. M., Cottrell, C. A., \& Schreindorfer, L. S. (2013). Construct validity of the need to belong scale: Mapping the nomological network. Journal of personality assessment, 95(6), 610-624. doi: 10.1080/00223891.2013.819511

Mardia, K. V. (1971). The effect of nonnormality on some multivariate tests and robustness to nonnormality in the linear model. Biometrika, 88(1), 105-121. doi: 10.1093/biomet/58.1.105

Nichols, A. L., \& Webster, G. D. (2013). The single item need to belong scale. Personality and Individual Differences, 55(2), 189-192. doi:http://dx.doi. org/10.1016/j.paid.2013.02.018

Patil, V. H., Surendra N. Singh, S. M., \& Donavan, T. (2007). Parallel analysis engine to aid determining number of factors to retain [Computer software]. Recuperado de http://smishra.faculty.ku.edu/parallelengine.htm

Pickett, C. L., Gardner, W. L., \& Knowles, M. (2004). Getting a cue: The need to belong and enhanced sensitivity to social cues. Personality and Social Psychology Bulletin, 30(9), 1095-1107. doi: 10.1177/0146167203262085

Pfundmair, M., Aydin, N., Du, H., Yeung, S., Frey, D., \& Graupmann, V. (2015). Exclude me if you can: cultural effects on the outcomes of social exclusion. Journal of Cross-Cultural Psychology. doi: 10.1177/0022022115571203 
Pfundmair, M., Graupmann, V., Frey, D., \& Aydin, N. (2015). The different behavioral intentions of collectivists and individualists in response to social exclusion. Personality and Social Psychology Bulletin, 41(3), 363-378. doi:10.1177/0146167214566186

Sampaio, L. R., Guimarães, P. R. B., dos Santos Camino, C. P., Formiga, N. S., \& Menezes, I. G.(2011). Estudos sobre a dimensionalidade da empatia: Tradução e adaptação do Interpersonal Reactivity Index (IRI). Psico, 42(1), 67-76. Recuperado de http:// revistaseletronicas.pucrs.br/ojs/index. $\mathrm{php} /$ revistapsico/article/view/6456

Schmitt, I. (2010). Normally I should belong to the others': Young people's gendered transcultural competences in creating belonging in
Germany and Canada. Childhood, 17(2), 163-180. doi: $10.1177 / 0907568210365643$

Tilvis, R. S., Routasalo, P., Karppinen, H., Strandberg, T. E., Kautiainen, H., \& Pitkala, K. H. (2012). Social isolation, social activity and loneliness as survival indicators in old age; a nationwide survey with a 7-year follow-up. European Geriatric Medicine, 3(1), 18-22. doi: 10.1016/j.eurger.2011.08.004

Waytz, A., \& Epley, N. (2012). Social connection enables dehumanization. Journal of Experimental Social Psychology, 48(1). doi:10.1016/j.jesp.2011.07.012

Recebido em: 08/06/2015 Reformulado em: 19/08/2015 Aprovado em: 27/08/2015

Nota dos autores:

Financiamento do CNPq por meio de bolsa de iniciação científica (PROIC-UnB) do primeiro autor e bolsa produtividade (PQ) do segundo autor e do Edital Universal do CNPq (478285/2013-2).

Sobre os autores:

Camila Azevedo Gastal é aluna de graduação do Instituto de Psicologia (IP) da Universidade de Brasília (Unb) e bolsista de Iniciação Científica do CNPq, desenvolvendo atividades no Laboratório de Psicologia Social (LAPSOCIAL) da Unb.

E-mail: camila.gastal@gmail.com

Ronaldo Pilati possui doutorado em Psicologia pela Universidade de Brasília; é professor adjunto do Departamento de Psicologia Social e do Trabalho (PST) e do Programa de Pós-Graduação em Psicologia Social, do Trabalho e das Organizações (PSTO) da UnB.

E-mail: rpilati@gmail.com

Contato com os autores:

Camila Azevedo Gastal

Universidade de Brasília

Laboratório de Psicologia Social

ICC Sul, sala A1-116

CEP: 70900-100

Brasília-DF, Brasil

Fone: (061) 3107-6874

Email: camila.gastal@gmail.com 